\title{
CATÁlOGO DE LÍQUENES DE ASTURIAS
}

\author{
Fernando DE LA TORRE FERNÁNDEZ y Mª Carmen FERNÁNDEZ ORDÓÑEZ
}

\begin{abstract}
RESUMEN. Catálogo de líquenes de Asturias. Catálogo de los líquenes de Asturias en el que se recogen 340 táxones en 180 localidades, provenientes de 2133 citas. Se incluyen datos procedentes tanto de referencias bibliográficas como de la revisión del Herbario FCO Lich.
\end{abstract}

Palabras clave. Catálogo, Liquen, Bibliografía, Asturias, España.

ABSTRACT. Lichen checklist of Asturias. The lichen checklist of Asturias collects 340 taxa in 180 places, from 2133 mentions. Data not only from bibliographic sources but also from the revision of the Herbarium FCO Lich. are included.

Key words. Checklist, Lichen, Bibliography, Asturias, Spain.

\section{INTRODUCCIÓN}

Para comenzar a hablar de la liquenología en Asturias debemos remontarnos a 1803 cuando Cavanilles publica, en la "Gaceta de Madrid", el artículo "Noticias del descubrimiento del liquen islándico en Asturias" -Cetraria islandica (L.) Ach.-. Más adelante Colmeiro (1889) recoge, además de sus propias aportaciones, citas de otros naturalistas, como Lagasca, Durieu de Maisonneuve, Pastor López y Lange, tomadas en sus itinerarios botánicos por la región. Trabajos destacables son también los desarrollados por Lázaro e Ibiza (1898) y Navas (1901). Entre 1926 y 1935 los esposos Allorge llevan a cabo campañas briófitas en España y, aunque su objetivo era la recopilación de briófitos, se recolectaron algunos líquenes determinados y publicados más tarde por
Abbayes (1945). Junto con Santesson (1960) y Ottonson (1968) destacan los trabajos de Vázquez, tanto su Memoria de Licenciatura (presentada en 1978) "Aportaciones al estudio de la flora liquénica epífita de las principales formaciones arboladas de Asturias y NE de León" como los realizados con Crespo y Barreno, entre los que cabe destacar Vázquez y Crespo (1978), que sienta las bases para el catálogo liquénico de la región. Esta labor florística y recopilatoria se continua en 1996 con la presentación de la Memoria de Licenciatura de Fernando de la Torre "Flora liquénica de Asturias”, donde se presentan las novedades y se adaptan o actualizan los nombres. El presente trabajo contiene la información básica del anterior, junto con la de trabajos recientes, como Renobales (1996) y Martínez Moreno (1999). 


\section{MATERIAL Y MÉTODOS}

Para la elaboración del catálogo se ha revisado la bibliografía que hace mención a las citas liquénicas en la región asturiana (tab. 1), desde Colmeiro (1889) hasta Martínez Moreno (1999). Por otro lado, se han incluido datos procedentes de la revisión y el estudio del Herbario de la Facultad de Ciencias de Oviedo (FCO Lich.), donde han sido y se encuentran depositados los pliegos resultantes de nuestras propias recolecciones.

La nomenclatura seguida en el catálogo es la propuesta por Purvis et al. (1992). Para la comprobación de los sinónimos que no figuran en esa obra se han seguido, según los casos, los criterios de Clauzade \& Roux (1985), Llimona (1991), Nimis (1993), Ozenda \& Clauzade (1970) o Poelt (1969).

En la tabla 1 se recogen los códigos que se asignan a los distintos orígenes de los datos (referencias bibliógráficas o pliegos del Herbario FCO Lich.) del catálogo.

Las localidades (Anexo I) en las que se cita cada especie, se indican con números detrás de las referencias bibliográficas; junto al número de la localidad aparecen, separados por un punto, el concejo, la descripción de la localidad, su altitud (msm) y las coordenadas

\begin{tabular}{ll}
\hline A Abbayes (1945) & N Martínez Moreno (1999) \\
B Barreno y Vázquez (1981) & $\tilde{N}$ Martínez y Burgaz (1993) \\
C Boom, van der & O Navas (1901) \\
$\quad$ \& Gómez-Bolea (1991) & P Ottonson (1968) \\
D Burgaz \& Athi (1992) & Q Renobales (1996) \\
E Burgaz \& Athi (1994) & R Santesson (1960) \\
F Colmeiro (1889) & S Timdal (1991) \\
G Crespo et al.(1979) & T Vázquez (Mem. Licenciatura*) \\
H Degelius (1954) & U Vázquez (1978) \\
I Dizerbo (1956) & V Vázquez (1981a) \\
J Egea (1984) & W Vázquez (1981b) \\
K Jauregui (1958) & X Vázquez y Crespo (1978) \\
L Kurokawa (1962) & Y Vitikainen (1980) \\
M Lazaro e Ibiza (1898) & Z Herbario FCO Lich. \\
\hline
\end{tabular}

Tabla 1. Relación de códigos y orígenes de datos incluidos en el catálogo. Relation between codes and data sources. (* Véase Introducción).
UTM; a continuación y entre paréntesis figura el número total de táxones que aparecen en la misma.

\section{RESULTADOS}

El catálogo se presenta ordenado taxonómicamente según Purvis et al. (l.c.). Los 340 táxones incluidos aparecen agrupados en 15 órdenes, 44 familias y 99 géneros.

Para cada tax on y entre paréntesis se indica el número total de localidades donde ha sido citado en la región; seguidamente se indica el origen de los datos (tab. 1) con las localidades que han sido recogidas en el mismo (fig. 1). Si procede, aparecerán diferentes códigos (que indican datos de procedencias distintas) separados por punto y coma.

\section{ASCOMYCOTINA}

ARTHONIALES Hessen ex D. Hawsw. \& O. Eriksson (1986)

Arthoniaceae Reichemb. (1941)

Arthonia Ach. (1806)

A. cinnabarina (DC.) Wallr. (9): T 1, 17, 132; V 42; W 174, 177; X 1, 17, 132.

A. elegans (Ach.) Almq. (5): T 78, 79; W $176 ;$ X 78, 79.

A. epiphyscia Nyl. (1): R 150.

A. fuliginosa (Turn et Borrer) Flot. (3): V 26, 42, 58 .

A. radiata (Pers.) Ach. (28): T 1, 18, 23, 76, 78, 88, 130, 132; V 26, 31, 42, 57, 58, 59, $60 ; \mathbf{W} 174,176,177 ; \mathbf{X} 1,18,23,66,76$, 78, 88, 129, 130, 132.

Chrysotricaceae Zahlbr. (1905)

Chrysotrix Mont. (1952)

C. candelaris (L.) Laundon (7): W 177; X 1, 65, 66, 79, 130, 152.

CALICIALES C. Bessey (1907)

Caliciaceae Chev. (1826)

Cyphelium Ach. (1815)

C. sessile (Pers.) Trevisan (2): T 152; X 17.

Coniocybaceae Reichemb. (1857)

Chaenotheca (Th. Fr.) Th. Fr. (1860)

C. trichialis (Ach.) Th. Fr. (2): C 162; M 127. 
Sphaerophoraceae Fr. (1831)

Sphaerophorus Pers. (1794)

S. globosus (Huds.) Vainio (19): F 72, 138; P 9; T 68, 88, 118, 122, 142, 152; W 179; X 68, 88, 118, 122, 141, 142, 152; Z 118, 141.

S. globosus var. curtus (Hook. et Tatl.) Zahlbr. (2): X 68, 118.

DOTHIDEALES Lindau (1897)

Arthopyreniaceae W. Watson (1929)

Arthopyrenia Massal. (1952)

A. punctiformis Massal. (7): V 25, 26, 31, $42,43,58,60$.

Leptorhapis Körber (1855)

L. epidermitis (Ach.) Th. Fr. (2): T 18; X 18.

GRAPHIDALES C. Bessey (1907)

Graphidaceae Dumort. (1822)

Graphis Adams (1763)

G. elegans (Borrer ex Sm.) Ach. (1): C 162.

G. scripta (L.) Ach. (25): T 18, 23, 65, 78, $80,88,129,132$; V 26, 42, 58; W 174, 176,$177 ; \mathbf{X} 18,23,65,78,80,88,129$, 132; Z 14, 118, 124.

Phaeographis Müll. Arg. (1882)

P. dendritica (Ach.) Müll. Arg. (16): T 2, 17, 18, 65, 130, 132; V 26, 42, 59; W 174, 176, 177; X 2, 18, 65, 132.

P. lyellii (Sm.) Zahlbr. (9): T 2, 18, 129, 130; X 2, 18, 65, 66, 129.

Thelotremataceae (Nyl.) Stizenb. (1862)

Diploschistes Norman (1853)

D. scruposus (Schreber) Norman var. bryophylus (Ehrh. ex Ach.) Müll. Arg. (5): U 86, 121, 151; W 172, 173.

Thelotrema Ach. (1803)

T. lepadinum (Ach.) Ach. (5): T 68, 122; W $179 ;$ X 68, 122.

GYALECTALES Henssen ex D. Hawksw. \& O. Eriksson (1986)

Gyalectaceae (Massal.) Stizenb. (1862)

? Belonia Körber ex Nyl. (1952)

B. russula Körber ex Nyl. (6): B 4, 5, 6, 8, $9,15$.

LECANORALES Nannf. (1932)

Alectoriaceae (Hue) Tomas. (1949)

Alectoria Ach. (1810)

A. prolixa (Ach.) Nyl. (6): P 9; T 88, 152; X $68,88,141$.

A. sarmentosa (Ach.) Ach. (4): T 68, 152;
W $179 ;$ X 141.

Bryoria Brodo \& D. Hawksw. (1977)

B. chalybeiformis (L.) Brodo \& D. Hawksw. (2): U 89; W 171.

B. haynaldii (Gyeln.) Brodo \& D. Hawksw. (4): T 88; W 179; X 88; Z 140.

B. implexa (Hoffm.) Brodo \& D. Hawksw. (7): P 9; T 88, 118; W 179; X 88, 118; Z 118.

B. jubata (L.) Brodo \& D. Hawksw. (10): F 138; T 88, 118, 142, 152; W 179; X 9, 68, $118,142$.

Bacidiaceae W. Watson (1929)

Bacidia de Not. (1846)

B. naegelii (Hepp) Zahlbr. (1): V 60.

Lecania Massal. (1853)

L. atrynoides Knowles (1): C 13.

L. tenera (Nyl.) Crombie (1): C 13.

L. turicensis (Hepp) Müll. Arg. (1): C 13.

? Squamarina Poelt (1859)

S. cartilaginea (With.) P. James (2): F 135; W 0.

S. gypsacea (Sm.) Poelt (1): U 105.

Toninia Massal. (1856)

T. candida (Weber ex Wigg.) Th. Fr. (3): F 72; S 0; W 170.

T. plumbina (Anzi) Hafellner \& Timdal (1): R 153.

Candelariaceae Halukinen (1954)

Candelaria Massal. (1852)

C. concolor (Dickson) B. Stein (1): V 28.

C. xanthostigma (Ach.) Lettau (2): V 35, 48.

Catillariaceae Hafellner (1984)

Catillaria Massal. (1852)

C. chalybeia (Borrer) Massal. (1): C 13.

C. elachista (Körber) Vainio (1): C 165.

Cladoniaceae Zenker (1827)

Cladonia Hill ex P. Browle (1756)

C. arbuscula (Wallr.) Flotow (1): W 172.

C. caespiticia (Pers.) Flörke (2): C 165; E 150.

C. ciliata Stirton var. tenuis (Flörke) Athi (2): D 67; W 172.

C. coccifera (L.) Willd. (3): W 172; Z 117, 118.

C. coniocraea (Flörke) Sprengel (2): Z 118, 140.

C. convoluta (Lam.) Cout. (1): W 173.

C. cyathomorpha Stirton ex W. Watson (1): 
E 147

C. deformis (L.) Hoffm. (1): F 54.

C. digitata (L.) Hoffm. (1): W 172.

C. fimbriata (L.) Fr. (4): F 138; W 172; Z $14,140$.

C. floerkeana (Fr.) Flörke (1): Z 118.

C. furcata (Huds.) Schrader (3): E 147; W 172; Z 118.

C. gracilis (L.) Willd. (1): F 69.

C. incrassata Flörke (1): E 160.

C. mitis Sandst. (1): W 172.

C. ochroclora Flörke (1): E 160.

C. polydactyla (Flörke) Sprengel (1): E 160.

C. portentosa (Dufour) Coem. (2): W 172; Z 118 .

C. pyxidata (L.) Hoffm. (5): F 138; W 172, 173; Z 115, 141.

C. ramulosa (With.) Laundon (2): E 160; Z 140.

C. rangiferina (L.) Weber ex Wigg. (2): F 138; W 172.

C. rangiformis Hoffm. (1): W 173.

C. squamosa (Scop.) Hoffm. (1): E 160.

C. uncialis (L.) Weber ex Wigg. (2): W 172; Z 117

Pycnothelia Dufour (1821)

P. papillaria Dufour (1): W 172.

Collemataceae Zenker (1827)

Collema Weber ex Wigg. (1780)

C. crispum (Huds.) Weber ex Wigg. (1): H 0 .

C. flaccidum (Ach.) Ach. (1): H 0.

C. furfuraceum (Arnold) Du Rietz (5): T 88, 152; W 179; X 88, 152.

C. nigrescens (Huds.) DC. (11): T 88, 118, 129, 141, 142; W 179; X 88, 118, 129. $141,142$.

C. subfurvum (Müll. Arg.) Degel (2): R 136,153

Leptogium (Ach.) Gray (1821)

L. brebissonii Mont. (2): T 152; X 152.

L. lichenoides (L.) Zahlbr. (2): T 88; X 88.

L. saturninum (Dickson) Nyl. (2): T 152; W 179.

Hymeneliaceae Körber (1855)

Aspicilia Massal. (1852)

A. calcarea (L.) Mudd. (2): U 92; W 170.

Lecanoraceae Körber (1854)

Lecanora Ach. (1810)

L. agardhiana Ach. (1): C 13.

L. allophana (Ach.) Röhl. (29): T 2, 17, 18,
$68,79,88,129,132 ; \mathrm{V} 25,26,27,31,42$, $46,57,58,59,60 ; \mathbf{W} 174,177,179 ; \mathbf{X} 2$, 17, 18, 68, 79, 88, 129, 132.

L. carpinea (L.) Vainio (7): T $68,88,152$; W 179; X 68, 88, 152.

L. chlarona (Ach.) Nyl. (2): T 68; X 68.

L. chlarotera Nyl. (24): T 2, 18, 78, 120, 129,$132 ; \mathbf{V} 25,26,27,28,31,42,43,50$, $59,60,61 ; W 176 ;$ X 2, 18, 78, 120, 129 , 132.

L. chlarotera f. meridionalis (H. Magn.) Ozenda \& Clauzade (1); T 68.

L. confusa Almb. (1): C 162.

L. conizaea (Ach.) Nyl. (2): T 1; X 1.

L. conizaeoides Nyl. ex Crombie (32): T 1, $16,21,65,66,80,131 ; \mathrm{V} 28,30,31,32$, $34,36,37,38,39,40,41,42,45,46,48$, 60,$61 ; W 178 ; X 11,16,21,65,66,80$, 131.

L. crenulata Hook. (1): C 13.

L. glabrata (Ach.) Malme (7): T 68, 88, 97; W 175; X 68, 88, 97.

L. hagenii (Ach.) Ach. (6): V 33, 34, 35, 37, 39,48 .

L. hagenii var. umbrina Ach. (3): V 28, 31 , 33.

L. helicopis (Wahlenb.) Ach. (1): C 13.

L. intumescens (Rebent.) Rabenh. (13): T $68,79,88,118,142,152$; W 179; X 68, $83,88,118,141,142$.

L. laevis Poelt (11): T 68, 79, 88, 142, 152; X 68, 79, 88, 141, 142, 152.

L. muralis (Schreber) Rabenh. (1): W 170.

L. muralis var. versicolor (Pers.) Tuck. (3): U 92, 114, 123.

L. pallida (Schreber) Rabenh. (17): T 1, 2, $16,17,18,79,83,152$; W 177; X 1, 2, 16, $17,18,79,83,141$.

L. pruinosa Chaub. (1): C 13.

L. subrugosa Nyl. (6): T 68, 118, 152; X 68, $118,152$.

Lecidella Körber (1855)

L. elaeochroma (Ach.) M. Choisy (42): T 2, $17,18,68,78,79,83,88,130,131,141$; V $25,26,27,31,34,37,42,46,48,60$, $61 ; \mathbf{W} 174,175,176,177 ; \mathbf{X} 2,12,17,18$, $23,68,78,79,80,83,88,129,130,131$, $132,152$.

Protoparmelia M. Choisy (1929)

P. badia (Hoffm.) Hafelllner (1): U 92. 
Tephromela M. Choisy (1929)

T. atra (Huds.) Ach. (10): T 79, 80, 83; V 25 ; W 171, 174, 179; X 79, 80, 83.

Lecideaceae Chev. (1826)

Hypocenomyce M. Choisy (1951)

H. caradocensis (Leighton ex Nyl.) P. James

\& G. Schneider (1): C 162.

H. scalaris (Ach. ex Lilj.) M. Choisy (1): C 165.

Lecidea Ach. (1803)

L. botryosa (Fr.) Th. Fr. (6): T 16, 129, 141; X 16, 129, 152.

L. hypnorum Lib. (1): C 165.

L. lapicida (Ach.) Ach. (2): U 89; W 171.

L. parasema (Ach.) Ach. (9): T 77, 97, 120, 142; W 175; X 77, 97, 120, 142.

L. stigmatea (Ach.) Hertel \& Leuckert $\mathrm{f}$. egena (Krempelh.) H. Magn. (2): U 92; W 170.

Megalosporaceae Vêzda ex Hafellner \& Bellem. (1982)

Megalospora Meyen (1843)

M. tuberculosa (Fée) Sipman (1): M 127.

Micareaceae Vêzda ex Hafellner \& Bellem. (1984)

Micarea Fr. (1825)

M. adnata Coppins (1): C 165.

M. melaena (Nyl.) Hedl. (1): C 165.

M. peliocarpa (Anzi) Coppins \& R. Sant. (1): C 13.

M. prasina $\mathrm{Fr}$. (1): C 165.

Mycoblastaceae Hafellner (1984)

Mycoblastus Norman (1852)

M. sanguinarius (L.) Norman (3): T 68; W $179 ; \mathbf{X} 68$.

Pannariaceae Tuck. (1872)

Degelia Arvidsson \& Galloway (1981)

D. atlantica (Degel.) P. Jørg. \& P. James (8): T 118, 141, 152; X 68, 76, 118, 141, 152.

D. plumbea (Lightf.) P. Jørg. \& P. James (10): R 150, 153; T 68, 152; W 179; X 68, $76,141,152 ; \mathbf{Z} 141$.

Pannaria Delise ex Bory (1828)

P. mediterranea $\mathrm{C}$. Tav. (5): T 68, 152; W 179; X 68, 152.

P. rubiginosa (Ach.) Bory (2): W 179; X 68.

Psoroma (Ach.) Michaux (1803)

P. hypnorum (Vahl.) Gray (2): U 95; W 172.
Parmeliaceae Zenker (1827)

Cetraria Ach. (1803)

C. chlorophylla (Willd.) Vainio (2): W 179; $\mathrm{X} 68$.

C. commixta (Nyl.) Th. Fr. (2): U 95; W 171.

C. ericetorum Opiz (2): U 90; W 172.

C. islandica (L.) Ach. (5): F 135, 137; W 172, 173; Z 99.

C. pinastri (Scop.) Gray (9): T 68, 118, 152; W 180; X 68, 86, 118, 141; Z 141.

C. sepincola (Ehrh.) Ach. (4): T 118; W $180 ; \mathbf{X} 86,118$

C. tilesii Ach. (1): U 163.

Cetrelia Culb. \& Culb. (1968)

C. cetrarioides (Del. ex Duby) Culb. \& C. Culb. (5): R 153; T 122, 141; W 179; Z 141.

C. olivetorum (Nyl.) Culb. \& C. Culb. (3): T 141; W 179; X 152.

Coelocaulon Link. (1833)

C. aculeatum (Schreber) Link (2): W 172, 173.

C. crespoae Barreno \& Vázquez (5): B 4, 5, $6,8,15$.

C. muricatum (Ach.) Laundon (2): U 95; W 172.

Cornicularia Hoffm. (1794)

C. normoerica (Gunn.) Du Rietz (2): F 69; W 171.

Evernia Ach. (1810)

E. prunastri (L.) Ach. (46): F 0; T 1, 2, 16, $17,18,68,76,78,79,80,83,88,118$, $122,131,142,145,152 ;$ V 57; W 174, $176,177,178,179 ;$ X 1, 2, 16, 17, 18, 68, $76,78,80,83,85,88,118,122,131,141$, 142, 145, 152; Z 118, 141.

Foraminella Fricke Meyer (1982)

F. ambigua (Wulfen) Fricke Meyer (3): T 118; W 180; X 118.

F. hyperopta (Ach.) Fricke Meyer (3): T 118; W 180; X 118.

Hypogymnia (Nyl.) Nyl. (1896)

H. intestiniformis (Vill.) Räsänen (1): W 171.

H. physodes (L.) Nyl. (30): F 0; P 9; T 16, $68,79,83,85,88,118,131,141,142$, 152; W 178, 179; X 1, 16, 68, 79, 83, 85, $88,118,122,131,141,142,152 ; \mathbf{Z} 118$, 141. 
H. tubulosa (Schaerer) Havaas (15): T 88, $118,122,131,141,145 ; \mathbf{W} 178,179 ; \mathbf{X}$ $68,118,122,131,141,145 ; \mathbf{Z} 141$.

Menegazzia Massal. (1854)

M. terebrata (Hoffm.) Massal. (3): T 152; W 179; X 152.

Parmelia Ach. (1903)

P. borreri (Sm.) Turner (2): M 150; 0158.

P. caperata (L.) Ach. (55): F 62; M 135; O 135, 158; T 1, 12, 16, 17, 18, 21, 23, 65, $66,80,83,85,122,129,130,131,132$, 152 ; V 26, 28, 30, 31, 33, 42, 57, 58, 59, 60; W 171, 174, 176, 177, 178; X 1, 12, $16,17,18,21,23,65,66,79,83,85,122$, 130, 131, 132, 152; Z 118.

P. conspersa (Ehrh. ex Ach.) Ach. (2): F 69; W 171.

P. crinita Ach. (4): O 158; T 152; W 179; X 152.

P. exasperata (Ach.) de Not. (5): T 88, 142; W 179; X 88, 142.

P. glabratula (Lamy) Nyl. (17): R 150, 153; T $18,68,83,88,118,141 ;$ W 177, 179; X $18,68,79,83,88,118,141$.

$P$. glabratula subsp. fuliginosa (Fr. ex Duby) Laundon (11): T 68, 80, 83, 85, 88; W 179; X 68, 80, 83, 85, 88 .

P. laevigata (Sm.) Ach. (2): T 18; X 18.

P. omphalodes (L.) Ach. (2): W 171; Z 118.

P. perforata Jacq. (1): O 150.

P. perlata (Huds.) Ach. (42): F 138; 0 63, 150, 158; T 1, 2, 12, 16, 17, 18, 79, 80, $129,130,131,132,152 ; \mathrm{V} 25,26,57,58$, 59,$60 ; \mathbf{W} 171,174,176,177,178 ; \mathbf{X} 1,2$, $12,16,17,18,79,80,130,131,132,152$; Z 14, 118.

P. pulla Ach. (1): W 171.

P. quercina (Willd.) Vainio (2): T 16; X 16.

P. reticulata Taylor (1): 0158.

P. saxatilis (L.) Ach. (21): F 72; K 69; M 150; O 150; T 68, 88, 118, 122, 142, 145, 152; W 171, 179; X 68, 88, 118, 122, 142, 145,$152 ; \mathbf{Z} 141$.

P. stuppea. Tayl. (2): T 152; X 152.

P. subaurifera Nyl. (31): T 17, 18, 68, 77, $79,83,88,97,118,130,141 ; \mathbf{V} 26,37$, $42,57,60$ : W 175, 177, 179; X 17, 18, 68, 76, 77, 79, 83, 88, 97, 118, 130, 152.

P. subrudecta Nyl. (6): V 26, 33, 34, 37, 57, 60.
P. sulcata Taylor (39): O 63; P 9; T 16, 68, $79,80,85 ; 88,118,122,131,142,145$, 152; V 25, 26, 27, 37, 42, 57, 58, 60; W 178,$179 ;$ X 16, 68, 79, 80, 83, 85, 88, $118,122,131,141,142,145,152 ; \mathrm{Z} 141$.

P. tiliacea (Hoffm.) Ach. (8): F 0; T 88, 122; W 171, 179; X 88, 122; Z 118.

P. tinctina Maheu \& A. Gillet (1): W 168.

Parmeliopsis (Nyl.) Nyl. (1866)

P. aleurites (Ach.) Nyl. (4): T 118; W 180; $\mathbf{X} 86,118$.

Platismatia Culb. \& C. Culb. (1868)

P. glauca (L.) Culb. \& C. Culb. (25): F 138; P 9; T 68, 79, 88, 118, 122, 131, 141, 142, 145, 152; W 178; X 68, 79, 88, 118, 122, $131,141,142,145,152 ; \mathbf{Z} 118,141$.

Pseudevernia Zopf (1903)

P. furfuracea (L.) Zopf (12): F 0; T 16, 68, $88,118,122,131,142,152$; W 178, 179; Z 141.

Usnea Dill. ex Adams. (1753)

$U$. ceratina Ach. subsp. incurvescens (Arnold.) Mot. (11): T 2, 16, 17, 18; W 177, 178; X 1, 2, 16, 17, 18.

U. comosa (Ach.) Röhl. (10): T 68, 85, 88, 141; W 179; X 68, 85, 88, 141; Z 118.

U. dasypoga (Ach.) Röhl. (8): F 0; T 68, 85, 88; X 68, 85, 88; Z 118

U. dasypoga subsp. melanorrhiza (Britz.) Mot. var. plicata (L.) Cromb. (1): F 0.

U. florida (L.) Wigg. (13): F 150; T 68, 85, 88, 141, 152; W 179; X 68, 85, 88, 141, 152; Z 118.

U. fulvoreagens (Räsänen) Räsänen (4): T 68, 88; X 68, 88.

U. hirta (L.) Wigg. (3): F 56; T 68, 88.

U. longissima Ach. (3): P 10, 11; W 179.

U. rubiginea (Michx.) Massal. (12): T 1, 2, 16, 17, 18; W 177, 178: X 1, 2, 16, 17, 18.

Physciaceae Zahlbr. (1898)

Anaptychia Körber (1848)

A. ciliaris Körber ex Massal. (6): T 88, 118 ; W 179; X 88, 118; Z 118.

A. runcinata (With.) Laundon (2): U 19; W 168.

Buellia de Not. (1846)

B. disciformis (Fr.) Mudd (18): T 16, 23, $68,79,80,83,118,142,152 ; \mathbf{X} 16,23$, $68,79,80,83,118,141,142$.

B. griseovirens (Turner \& Borrer ex Sm.) 
Almb. (6): B 4, 5, 6, 8, 9, 15.

B. pseudosaxatilis Samp. (1): R 150.

B. punctata (Hoffm.) Massal. (21): T 79, $80,83,141$; V 25, 31, 33, 34, 36, 37, 39, 40, 41, 42, 48, 50, 60; X 79, 80, 83, 141.

Diploicia Massal. (1852)

D. canescens (Dickson) Massal. (16): G 47; U 19, 29, 46, 49, 52; V 26, 27, 31, 33, 34, 35, 37, 43, 48; W 171.

D. subcanescens (R.G. Wegner) Massal. (1): G 47 .

Diplotomma Flotow (1849)

D. ambiguum (Ach.) Flagey (1): C 13.

Heterodermia Trevisan (1868)

H. leucomelos (L.) Poelt (1): M 22.

H. obscurata (Nyl.) Trevisan (4): L 22; T 141; W 179; X 152.

Hyperphyscia Müll. Arg. (1894)

H. adglutinata (Flörke) Mayrh. \& Poelt (15): V 25, 30, 31, 34, 35, 36, 37, 39, 40, 41, $43,46,48,58,60$.

Phaeophyscia Moberg (1877)

P. ciliata (Hoffm.) Moberg (1): M 127.

P. orbicularis (Necker) Moberg (4): R 150; V 30, 35, 42.

Physcia (Schreber) Michaux (1803)

P. adscendes (Fr.) H. Olivier (39): T 77, 88, 97, 120, 122, 129, 141; V 25, 26, 27, 28, $30,31,33,34,36,37,39,41,42,46,48$, $50,57,58,59,60,61 ; \mathbf{W} 174,175,176 ; \mathbf{X}$ $68,77,88,97,120,122,129,152$.

P. aipolia (Ehrh. ex Humb.) Fürnrohr (8): T 78, 80, 129; W 174, 176; X 78, 80, 129.

$P$. caesia (Hoffm.) Fürnrohr (4): U 112, 121, 125; W 171.

P. clementei (Sm.) Maas Geest. (2): V 25, 26.

P. semipinnata (Gmelin) Moberg (12): F 0; T 77, 78, 97; V 43, 60, 61; W 175, 176; X $77,78,97$.

P. stellaris (L.) Nyl. (2): F $0 ; \mathbf{X} 78$.

P. tenella (Scop.) DC. (3): T 97; W 175; X 97.

P. tribacoides Nyl. (6): T 17; V 31, 34, 58, 60; X 17.

Physconia Poelt (1965)

P. distorta (With.) Laundon (9): T 88, 118 , 152; W 179; X 83, 88, 152; Z 118, 141.

P. farrea (Ach.) Poelt (3): T 118; W 179; X 118.
P. grisea (Lam.) Poelt (4): V 33, 34, 35, 37

P. muscigena (Ach.) Poelt (4): F 7; U 109, 112; W 173.

P. venusta (Ach.) Poelt (4): T 88; W 179; X 88; Z 118.

Rinodina (Ach.) Gray. (1753)

R. gennarii $\mathrm{Bagl}$. (1): C 13.

Psoraceae Zahlbr. (1898)

Protoblastenia (Zahlbr.) Steiner (1911)

P. calva (Dickson) Zahlbr. (2): U 104; W 170.

P. incrustans (DC.) Steiner (3): U 104, 113; W 170.

Psora Hoffm. (1796)

P. decipiens (Hedw.) Hoffm. (2): U 108; W 173.

P. lurida (Ach.) DC. (2): F 72; W 170.

Ramalinaceae Ag. (1821)

Ramalina Ach. (1810)

R. calicaris (L.) Fr. (8): T 83, 129, 152; W 177, 179; X 141; Z 118, 141.

R. farinacea (L.) Ach. (48): T 12, 16, 18, $68,76,78,79,80,83,85,88,97,118$, $122,129,142,145,152 ;$ V 26, 42, 58, 60; W 174, 175, 176, 177, 179; X 12, 16, 18, $68,76,78,79,80,83,85,88,97,118$, $122,129,141,142,145,152 ; \mathbf{Z} 118,141$.

R. fastigiata (Pers.) Ach. (9): F 0; T 79, 88, 118; W 179; X 85, 88, 118; Z 118.

$R$. fraxinea (L.) Ach. (2): F 0; Z 141.

R. pollinaria (Westr.) Ach. (3): F 0; $\mathbf{T} 79 ; \mathbf{X}$ 85.

R. polymorpha (Lilj.) Ach. (2): F 72, 138.

$R$. pyrifera (Nyl.) Mot. (1): P 9.

R. siliquosa (Hudson) A. L. Sm. (1): W 168.

R. thrausta (Ach.) Nyl. (3): T 68; W 179; X 68.

? Rhizocarpaceae M. Choisy ex Hafellner (1984)

Rhizocarpon Lam. ex DC. (1805)

R. alpicola (Anzi) Rabenh. (2): U 89; W 171.

R. effiguratum (Anzi) Th. Fr. (2): U 92; W 171.

R. geographicum (L.) DC. (1): W 171.

$R$. lecanorinum Anders (1): U 93.

R. umbilicatum (Ramond) Flagey (2): U 92; W 170.

Trapeliaceae M. Choisy ex Hertel (1970)

Trapelia M. Choisy (1929)

T. corticola Coppins \& P. James (1): C 162. 
T. involuta (Taylor) Hertel (1): C 13.

Trapeliopsis Hertel \& Schneid. (1980)

T. viridescens (Schrader) Coppins \& P. James (1): C 162.

Umbilicariaceae Chev. (1826)

Lasallia Merát (1821)

L. hispanica (Frey) A. Crespo \& G. Sancho (1): W 171

L. pustulata (L.) Mérat (2): F 72; W 171.

Umbilicaria Hoffm. (1789)

U. crustulosa (Ach.) Frey (1): W 171.

U. cylindrica (L.) Delise ex Duby (4): F 72; W 171; Z 64, 118.

U. deusta (L.) Baumg. (1): W 171.

U. hyperborea (Ach.) Hoffm. (1): W 171.

Genera inciertae

Lepraria Ach. (1803)

L. incana (L.) Ach. (11): V 27, 28, 31, 33, 34, 36, 37, 38, 40, 42, 43.

L. neglecta (Nyl.) Lettau (4): T 68, 79; X 68, 79.

Leprocaulon Nyl. ex Lamy (1878)

L. microscopicum (Vill.) Gams ex D. Hawskw. (12): V 27, 28, 30, 31, 33, 34, $36,37,38,40,42,43$.

LEOTIALES Carpenter (1988)

Baeomycetaceae Dumort (1829)

Baeomyces Pers. (1794)

B. roseus Pers. (1): W 172.

B. rufus (Huds.) Rebent. (4): U 19, 139, 141; W 172.

LICHINALES Henssen \& Büdel (1986)

Lichinaceae Nyl. (1854)

Lichina Agardh (1817)

L. confinis (Müller) Agardh (3): I 47, 52; W 169.

L. pygmaea (Lighft.) Agardh (5): F 54; I 24, 52, 134; Z 51 .

Zahlbrucknerella Herre (1963)

Z. calcarea (Herre) Herre (1): C 159.

OPEGRAPHALES M. Choisy ex D. Hawksw. \&

Eriksson (1986)

Opegraphaceae Stizenb. (1862)

Lecanactis Körber (1825)

L. abietina (Ach.) Körber (1): C 165.

L. lyncea (Sm.) Fr. (1): C 165.

L. premnea (Ach.) Arnold (1): C 165.

Opegrapha Humb. (1973)

O. atra Pers. (23): T 12, 23, 78, 79, 129 , 173; V 25, 26, 42, 46, 50, 57, 58, 59, 60;
W 174, 176; X 12, 23, 78, 79, 129, 132.

O. conferta Anzi (1): C 13.

O. phegospila Nyl. (2): V 31, 36.

O. viridis (Ach.) Nyl. (2): V 40, 42.

O. varia Pers. (2): T $68 ; \mathbf{X} 68$.

Roccellaceae Chev. (1826)

Roccella DC. (1805)

R. fuciformis (L.) DC. (2): F 20; W 168.

R. phycopsis Ach. (2): U 14; W 168.

OSTROPALES Nannf. (1932)

Stictidaceae Fr. (1849)

? Petractis Fr. (1846)

P. clausa (Hoffm.) Krempelh. (1): U 121.

PELTIGERALES W. Watson (1929)

Lobariaceae Chev. (1826)

Lobaria (Schreber) Hoffm. (1796)

L. amplissima (Scop.) Forss. (18): C 165; F 138; T 88, 118, 122, 142, 145, 152; W $179 ; \mathbf{X} 68,76,88,118,122,141,152 ; \mathbf{Z}$ $118,141$.

L. pulmonaria (L.) Hoffm. (31): C 165; F 0, 138; M 153; R 153; T 68, 76, 80, 85, 88, $118,122,141,142,145,152 ;$ W 179; X $68,76,80,83,85,88,118,122,141,142$, 145,$152 ; \mathbf{Z} 118,141$.

L. pulmonaria var. meridionalis (Vain.) Zahlbr. (5): T 145, 152; X 68, 145, 152.

L. scrobiculata (Scop.) DC. (12): F 0; P 9; T 88, 141, 152; W 179; X 68, 83, 118, 141, 152; Z 141 .

L. virens (With.) Laundon (5): C 165; M 127; T 68; W 179; X 68.

Sticta (Schreber) Ach. (1803)

S. fuliginosa (Hoffm.) Ach. (2): W 179; X 68.

S. sylvatica (Huds.) Ach. (1): T 68 .

Nephromataceae Wetm. ex J.C. David \& D. Hawksw. (1991)

Nephroma Ach. (1810)

N. laevigatum Ach. (17) M 150; P 9; T 68, 83, 88, 118, 141, 152; W 179; X 68, 76, 83, 88, 118, 141, 152; Z 141.

N. parile (Ach.) Ach. (7): T 88, 118, 152; W $179 ; \mathbf{X} 88,118,152$.

N. resupinatum (L.) Ach. (15): F 137; T 68, 83, 88, 118, 141, 152; W 179; X 68, 83, $88,118,141,152 ; \mathbf{Z} 141$.

Peltigeraceae Dumort. (1822)

Peltigera Willd. (1787)

P. canina (L.) Willd. (31): M 166; N 74, 81, 
$82,107,110,119,149,157 ; \mathbf{T} 68,76,79$, $88,118,122,141,145,152 ;$ W 172, 177 179; X 68, 76, 79, 88, 118, 122, 141, 145 , 152; Z 140 .

P. collina (Ach.) Schrader (16): N 68, 71, 82, 101, 148; T 88, 122, 141, 142, 152; W 179; X 68, 88, 141, 142, 152.

P. degenii Gyelnik (5): N 71, 75, 143; T $118 ; \mathbf{X} 118$.

P. didactyla (With.) Laundon var. extenuata (Nyl. ex Vainio) Goffinet \& Hastings (2): N 71, 82 .

P. elisabethae Gyelnik (3): N 87, 107, 161. P. horizontalis (Huds.) Baumg. (27): F 0; M 166; N 82, 101, 107, 116, 148, 149; T $68,76,79,88,122,141,142,152 ;$ W 177 , 179 ; X 68, 76, 79, 88, 122, 141, 142, 152; Z 140

P. hymenina (Ach.) Delise (9): N 3, 71, 75, 87, 118, 144, 149; Z 14, 118.

P. kristinssonii Vitik. (1): N 116.

P. lactucifolia (With.) Laundon (1): Y 0.

P. lepidophora (Nyl.) Bitter (1): N 158.

P. malacea (Ach.) Funck. (2): $\tilde{\mathbf{N}}$ 99; U 92.

P. membranacea (Ach.) Nyl. (11): N 70, 71 , $74,82,107,116,118,128,143,146 ; \mathbf{Z}$ 118.

P. monticola Vitik. (2): N 107, 157.

P. neckeri Hepp ex Müll. Arg. (2): N 82, 107.

P. neopolydactyla (Gyeln.) Gyeln. (2): N $143,146$.

P. polydactyla (Necker) Hoffm. (16): F 62, 138; N 158; T 68, 79, 88, 142; W 172, 177, 179; X 68, 76, 88, 142, 152; Z 141.

$P$. ponojensis Gyeln. (2): $\mathbf{N} 82,96$.

P. praetextata (Flörke ex Sommerf.) Zopf (18): N 71, 74, 75, 87, 94, 101, 116, 148, $150,157,158,164 ; \mathbf{R} 153 ; \mathbf{T} 88,152 ; \mathbf{W}$ $179 ; \mathbf{X} 88,152$.

P. rufescens (Weis) Humb. (13): F 166; $\mathbf{N}$ 82, 96, 107, 110, 118, 126; N 154, 157, 158; R 136; W 173; Z 118.

Solorina Ach. (1808)

S. bispora Nyl. var. macrospora (Harm.) H. Olivier (2): U 92; W 173.

S. saccata (L.) Ach. (5): F 72; R 153; W $173 ; \mathbf{Z ~ 1 4 0 , 1 5 0 .}$

? Placynthiaceae Dahl (1950)

Placynthium (Ach.) Gray (1821)
P. filiforme (Garov.) Choisy (1): C 155.

$P$. lismorense (Nyl. ex Crombie) Vainio (1): C 155.

P. nigrum (Huds.) Gray (2): U 121; W 170.

P. subradiatum (Nyl.) Arnold (1): C 155.

P. tremniacum (Massal.) Jatta (1): C 155.

PERTUSARIALES M. Choisy ex D. Hawksw. \& O.

Eriksson (1986)

Pertusariaceae Körber ex Körber (1855)

Ochrolechia Massal. (1852)

O. androgyna (Hoffm.) Arnold (3): T 152; W 179; X 141.

O. balcanica Vers. (3): T 152; W 179; X 152.

O. pallescens (L.) Massal. (1): T 152.

O. pallescens f. pulverulenta Vers. (1): $\mathbf{X}$ 141.

O. parella (L.) Massal. (4): F 0; U 19; W $171 ; \mathbf{Z} 118$

O. parella subsp. anomala (Harm.) Clauzade \& Roux (1): B 8.

O. subviridis (Høeg) Erichsen (2): T 122; X 152.

O. upsaliensis (L.) Massal. (3): U 91, 111; W 173

Pertusaria DC. (1805)

P. albescens (Huds.) M. Choisy \& Werner (3): T 152; W 179; X 152.

P. $\operatorname{amara}$ (Ach.) Nyl. (31): T 2, 17, 18, 76, $79,85,88,118,122,142,145,152$; W $174,177,179 ; \times 2,17,18,68,76,79,80$, $83,85,88,118,122,141,142,145,152$.

P. flavida (DC.) Laundon (10): T 79, 88, 130, 152; W 177, 179; X 18, 68, 79, 130.

P. gallica B. de Lesd. (1): G 47.

P. glomulifera (Turner) Massal. (27): T 66, $68,79,80,83,85,88,118,122,129,130$, 142,$152 ; \mathbf{X} 66,68,79,80,83,85,88$, $118,122,129,130,141,142,152$.

P. hemisphaerica (Flörke) Erichsen (8): T 79, 83, 88, 152; X 79, 83, 88, 141.

P. heterochroa (Müll. Arg.) Erichsen (10): T 79; V 26, 31, 42, 57, 58, 59, 60; W 179; X 79.

P. laevigata (Nyl.) Arnold. (2): T 18; X 18.

P. lecanorodes Erichsen (11): T 2, 68, 118 , 142, 152; W 179; X 2, 68, 118, 141, 142.

P. leioplaca DC. (4): T 76, 122; X 76, 122.

$P$. leprarioides Erichsen (1): $\mathbf{R} 150$.

P. leptospora Nitschke (2): T 152; X 152. 
P. leucosora Nyl. (5): T 88, 141, 142; X 88, 142.

P. mammosa Harm. (2): T 80; X 80.

P. multipuncta (Turner) Nyl. (3): T $68 ; \mathbf{W}$ $179 ; \mathbf{X} 68$.

P. pertusa (Weigel.) Tuck. (5): T 68, 118; W 179; X 68, 118.

P. pustulata (Ach.) Duby (3): T 66; W 177; X 66.

PYRENULALES Fink ex D. Hawksw. \& O. Eriksson (1986)

Pyrenulaceae Rabenh (1870)

Acrocordia Massal. (1854)

A. conoidea (Fr.) Körber (2): U 66; W 170.

Pyrenula Massal. (1852)

P. nitida (Weigel) Ach. (3): T 152; W 179;

X 152.

Trichotheliaceae (Müll. Arg.) Bitter \& Schill. (1927)

Porina Müll. Arg. (1883)

P. carpinea (Pers.) Zahlbr. (2): T 18; X 18.

P. leptalea (Dur. \& Mont.) A. L. Sm. (2): T 17; X 17.

TELOSCHISTALES D. Hawksw. \& O. Eriksson (1986)

Teloschistaceae Zahlbr. (1898)

Caloplaca Th. Fr. (1860)

C. arenaria (Pers.) Müll. Arg. (1): J 73.

C. biatorina (Massal.) Steiner (3): J 156; U 121; W 170.

C. chalybaea (Fr.) Müll. Arg. (1): J 156.

C. cirrochroa (Ach.) Th. Fr. (2): C 13; J 150.

C. citrina (Hoffm.) Th. Fr. (2): V 42, 48.

C. ferruginea (Huds.) Th. Fr. (13): T 77, 79, $80,83,141$; W 175, 179; X 68, 77, 79, 80, 83,141 .

C. flavescens (Huds.) Laundon (3): U 19, 53; W 170.

C. lucifuga Thor (1): C 162.

C. luteoalba (Turner) Th. Fr. (1): T 85.

C. marina (Wedd.) Zahlbr. ex Du Rietz (2): I 47; W 169.

C. microthallina (Wedd.) Zahlbr. (1): U 19.

C. ochracea (Schaerer) Flagey (1): J 150.

C. saxicola (Hoffm.) Nordin (1): J 156.

Fulgensia Massal. \& de Not. (1972)

F. fulgens (Swartz) Elenkin (2): U 98; W 173.

Xanthoria (Fr.) Th. Fr. (1880)
X. calcicola Oxner (3): U 112; W 169, 170.

X. candelaria $($ L.) Th. Fr. (2): T 118; X 118.

X. elegans (Link.) Th. Fr. (5): U 100, 102, 112, 125; W 170.

$X$. parietina (L.) Th. Fr. (41): F 0; I 47, 52; T 77, 78, 97, 120, 122, 129, 132, 152; V $25,26,28,30,33,34,35,36,37,39,41$, $42,46,48,57,58,59,60,61$; W 169, 175; X 77, 78, 97, 120, 122, 129, 132, 152; Z 118.

VERRUCARIALES Mattick ex D. Hawksw. \& O. Eriksson (1986)

Verrucariaceae Zanker (1827)

Agonimia Zahlbr. (1909)

A. tristicula (Nyl.) Zahlbr. (1): C 155.

Dermatocarpon Eschw. (1924)

D. luridum (With.) Laundon (3): F 72; U 167; W 171.

D. miniatum (L.) Mann. (3): A 0; F 72; W 170.

D. monstruosum (Schaer.) Vain. (2): U 92; W 170.

Polyblastia Massal. (1852)

P. cupularis Massal. (1): Q 106.

Staurothele Norman (1853)

S. fissa (Taylor) Zwackh (2): U 84; W 171.

Thelidium Massal. (1855)

T. papulare (Fr.) Arnold (1): Q 106.

Verrucaria Schrader (1794)

V. marmorea (Scop.) Arn. (3): U 92, 114; W 170.

V. maura Wahlenb. (6): I 24, 47, 52, 133, 134; W 169.

V. nigrescens Pers. (2): U 92; W 170.

V. parmigera Steiner (4): U 66, 114, 121; W 170.

V. parmigerella Zahlbr. (2): U 66; W 170.

V. symbalana Nyl. (1): W 169.

\section{ORDEN INCIERTO}

Phlyctidaceae Poelt ex J.C. David \& D. Hawksw. (1991)

Phlyctis Wallr. (1825)

P. argena (Sprengel) Flotow (7): T 88, 97, 132; W 179; X 88, 97, 132.

\section{GENERO INCIERTO}

Normandina Nyl. (1855) 
N. pulchella (Borrer) Nyl. (9): T 85; V 31 , 42, 43; W 177, 179; X 68, 76, 85.

\section{ESPECIES DUDOSAS}

\section{Lecanora (Lecanoraceae)}

L. haematomma Ach. (2): F 72, 138.

Lecidea (Lecideaceae)

L. albo-caerulescens Ach. (1): F 69.

\section{ANEXO I}

Lista de localidades (fig. 1); entre paréntesis se indica el número de táxones citados en cada localidad.

$0 . \quad$ Sin localidad precisa.

1. Tapia. Río Porcia. 5m. 29TPJ62 (21).

2. Tapia. La Barrosa. 50m. 29TPJ7125 (24).

3. Villayón. Castañedo en Barandón. $950 \mathrm{~m}$. 29TPJ80 (1).
4. Illano. San Esteban de los Buitres. $450 \mathrm{~m}$. 29TPH7598 (3).

5. San Martín de Oscos. San Martín de Oscos. 700m. 29TPH6592 (3).

6. Pesoz. San Pedro de Agüeria. $500 \mathrm{~m}$. 29TPH6990 (3).

7. Grandas de Salime. Peñafurada. $700 \mathrm{~m}$. 29TPH6879 (1).

8. Allande. Cornollo. 500m. 29TPH7782 (4).

9. Ibias. San Antolín de Ibias. 310m. 29TPH7367 (12).

10. Ibias. Valdebueyes. $700 \mathrm{~m} .27 \mathrm{TPH} 8268$ (1).

11. Ibias. Omente. 400m. 29TPH8461 (1).

12. Orillas del Río Negro. 50m. 29TPJ9719 (9).

13. Valdés. $2 \mathrm{~km}$ NE de Luarca, cara $\mathrm{N}$ de un roquedo costero. $2-50 \mathrm{~m}$. 29TPJ92 (14).

14. Valdés. Luarca. 30m. 29TPJ9922 (5).

15. Valdés. Busto. 50m. 29TQJ0525 (3).

16. Valdés. Carcedo. 150m. 29TQJ0918 (27).

17. Valdés. Arcallana. 200m. 29TQJ1722 (28).

18. Valdés. Entre Pontigón y Ayones. $240 \mathrm{~m}$. 29TQJ01 (43).

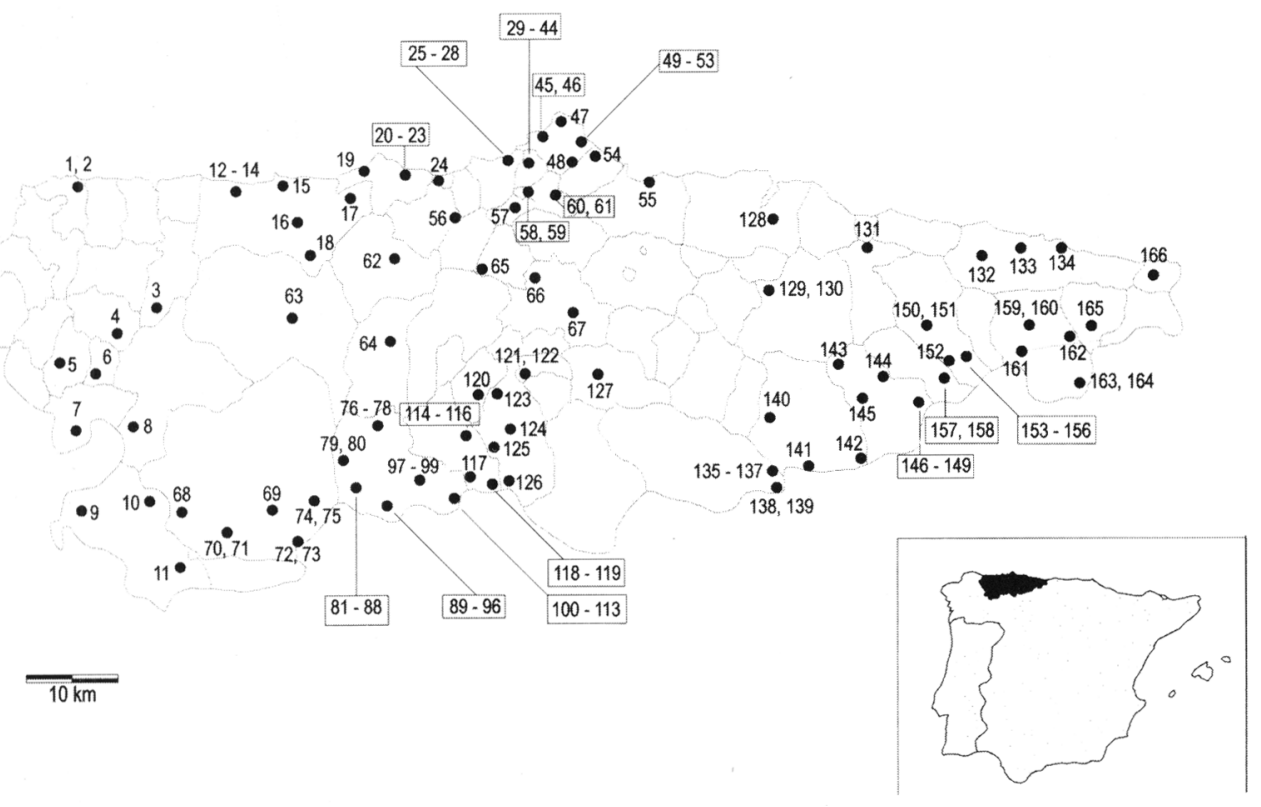

Figura 1. Posición aproximada de las localidades. Approximate position of the localities. 
19. Cudillero. Novellana. 50m. 29TQJ1927 (6).

20. Cudillero. Concha de Artedo. 5m. 29TQJ22 (1).

21. Cudillero. Lamuño. 10m. 29TQJ2627 (4).

22. Cudillero. Cudillero. 50m. 29TQJ3026 (2).

23. Cudillero. San Pedro de la Ribera. $10 \mathrm{~m}$. 29TQJ2428 (11).

24. Muros del Nalón. Aguilar. 5m. 29TQJ3226 (2).

25. Castrillón. Piedras Blancas. 5m. 30TTP2927 (13).

26. Castrillón. El Cuadro. 100m. 30TTP5923 (20).

27. Castrillón. Arnao. 5m. 30TTP5929 (8).

28. Castrillón. Salinas. 5m. 30TTP6029 (9).

29. Avilés. Avilés. 20m. 30TTP62 (1).

30. La Carriona. 50m. 30TTP6225 (7).

31. Avilés. Gaxín. 30m. 30TTP6227 (18).

32. Avilés. San Juan de Nieva. 5m. 30TTP6321 (1).

33. Avilés. Carbayedo. 15m. 30TTP6326 (11).

34. Avilés. Las Meanas. 5m. 30TTP6327 (13).

35. Avilés. Entrecarreteras. 10m. 30TTP6425 (7).

36. Avilés. Jardín de Cantos. 15m. 30TTP6426 (8).

37. Avilés. Parque de Ferrera. 10m. 30TTP6426 (14).

38. Avilés. Parque del Muelle. 5m. 30TTP6427 (3).

39. Avilés. Paseo de la Ría. 5m. 30TTP6428, 6429 (6).

40. Avilés. Villalegre. 25m. 30TTP6524 (6).

41. Avilés. Llaranes. 10m. 30TTP6526 (5).

42. Avilés. La Vallina. 50m. 30TTP6527 (25).

43. Avilés. Valliniello. 50m. 30TTP6528 (8).

44. Avilés. Tabiella. $90 \mathrm{~m}$. 30TTP6628 (1).

45. Gozón. Xagó. 5m. 30TTP63 (1).

46. Gozón. San Martín de Podes. 70m. 30TTP6633 (8).

47. Gozón. Cabo Peñas. 50m. 30TTP7037 (7).

48. Gozón. La Granda. 100m. 30TTP7027 (10).

49. Gozón. Biforco. 20m. 30TTP73 (1).

50. Gozón. Mangán. 15m. 30TTP73 (4).

51. Gozón. Bañugues. 5m. 30TTP7334 (1).

52. Gozón. Luanco. 15m. 30TTP7433 (5).

53. Gozón. Moniello. 15m. 30TTP7433 (1).

54. Carreño. Candás. 30m. 30 TTP7630 (2).

55. Gijón. Gijón. 20m. 30TTP82 (5).

56. Pravia. Pravia. 30m. 29TQJ3319 (1).

57. Illas. Caño. 100m. 30TTP6322 (12).

58. Corvera. San Juan de Villa. 100m. 30TTP6223 (15).
59. Corvera. Llandones. 30m. 30TTP6422 (10).

60. Corvera. Nubledo. 50m. 30TTP6723 (21).

61. Corvera. Trasona. 20m. 30TTP6726 (6).

62. Salas. Villamar. $300 \mathrm{~m}$. 29TQJ2310 (2).

63. Tineo. Tineo. 650m. 29TQJ0904 (2).

64. Belmonte de Miranda. Belmonte. $200 \mathrm{~m}$. 29TQH2596 (1).

65. Las Regueras. Entre la Arocha y Puerma. $75 \mathrm{~m}$. 29TQJ4009, 4109 (10).

66. Oviedo. Brañes. 100m. 30TTP6410 (14).

67. Oviedo. Oviedo. 300m. 30TTP60, 70 (1).

68. Cangas del Narcea. Bosque de Muniellos. 7001600m. 29TPH (107).

69. Cangas del Narcea. Monte al O del Naviego. 900-1700m. 29TQH (5).

70. Cangas del Narcea. Pueblo de Rengos. $1200 \mathrm{~m}$. 29TPH9366 (1).

71. Cangas del Narcea. Moncó (Sierra del Rañadoiro). 1150m. 29TPH9367 (6).

72. Cangas del Narcea. Pico de Arbas. $1800 \mathrm{~m}$. 29TQH06 (11).

73. Cangas del Narcea. Laguna de Arbás (Puerto de Leitariegos). 1650m. 29TQH0963, 1063 (1).

74. Cangas del Narcea. Río Cibea (Genestoso). 1200m. 29TQH1170 (3).

75. Cangas del Narcea. Genestoso. $1200 \mathrm{~m}$. 29TQH1271 (3).

76. Somiedo. Entre Aguasmestas y La Riera. $500 \mathrm{~m}$. 29TQH28 (23).

77. Somiedo. Entre La Malva y La Riera. $300 \mathrm{~m}$. 29TQH28 (12).

78. Somiedo. La Malva. 400m. 29TQH28 (23).

79. Somiedo. Entre Pigüeña y La Rebollada. $700 \mathrm{~m}$. 29TQH17 (56).

80. Somiedo. Pigüeña. 700m. 29TQH1779 (33).

81. Somiedo. Brañas de la Pornacal. $1000 \mathrm{~m}$. 29TQH1773 (1).

82. Somiedo. Río Pigüeña (Villar de Vildas). 1050m. 29TQH1775 (8).

83. Somiedo. La Rebollada. $700 \mathrm{~m}$. 29TQH1678 (41).

84. Somiedo. Arroyos del Lago Bueno y de la Vega de Penouta. 1490m. 29TQH17 (1).

85. Somiedo. Entre Cores y Villar de Vildas. $700 \mathrm{~m}$. 29TQH17 (28).

86. Somiedo. Sierra del Páramo. 1800m. 29TQH17 (4).

87. Somiedo. Villar de Vildas. Subida a la Pornacal. 1050m. 29TQH 17 (3)

88. Somiedo. La Pornacal. 300m. 29TQH1871 
(106).

89. Somiedo. Base del Cornón. 1900m. 29TQH16 (3).

90. Somiedo. Laderas del Cornón. 2000m. 29TQH16 (1).

91. Somiedo. Ladera N del Penouta. $1800 \mathrm{~m}$. 29TQH17 (1).

92. Somiedo. Laderas del Penouta. $1700 \mathrm{~m}$. 29TQH17 (11).

93. Somiedo. Cumbre del Cornón. 2190m. 29TQH1967 (1).

94. Somiedo. Río Pigüeña (Brañas de los Cuartos). 1240m. 29TQH1971 (1).

95. Somiedo. Ladera de Vega Cimera. $1500 \mathrm{~m}$. 29TQH26 (3).

96. Somiedo. Puerto de Somiedo. 1450m. 29TQH26 (2).

97. Somiedo. Pola de Somiedo. 700m. 29TQH2374 (18).

98. Somiedo. Cercanías de Pola de Somiedo. 900m. 29TQH27 (1).

99. Somiedo. Somiedo. 800m. 29TQH28 (2).

100. Somiedo. Cumbre de Peña Blanca. $1900 \mathrm{~m}$. 29TQH2269 (1).

101. Somiedo. Fuente de Vega Ventana. $1300 \mathrm{~m}$. 29TQH2468 (3).

102. Somiedo. Sta. María del Puerto. $1500 \mathrm{~m}$. 29TQH2567 (1).

103. Somiedo. Cercanías de la Peral. $1250 \mathrm{~m}$. 29TQH26 (1).

104. Somiedo. Laderas de Peña Blanca. $1800 \mathrm{~m}$. 29TQH26 (2).

105. Somiedo. Cercanías del Valle del Lago. 1700m. 29TQH27 (1).

106. Somiedo. Murias Longas. 1600m. 29TQH27 (2).

107. Somiedo. Valle de Lago. 1500m. 29TQH2972, 3072, 3171 (7).

108. Somiedo. Mortera del Coto. $1900 \mathrm{~m}$. 29TQH3369 (1).

109. Somiedo. Lago del Valle. 1650m. 29TQH3369 (1).

110. Somiedo. Lago del Valle. 1700m. 29TQH3370 (2).

111. Somiedo. Peña Orniz. 2000m. 29TQH3467 (1).

112. Somiedo. Orillas del lago La Calabazosa. 1700m. 29TQH3570, 3670 (4).

113. Somiedo. Laderas de Peña Orniz. 1900m. 29TQH36 (1).

114. Teverga. Peña Sobia. $1100 \mathrm{~m}$. 29TQH38 (3).
115. Teverga. Carretera Pto. Ventana (Km 16). 700m. 29TQH3979 (1).

116. Teverga. Páramo. Río Teverga. $1400 \mathrm{~m}$. 29TQH4476 (4).

117. Teverga. Bosque de Villanueva. $1200 \mathrm{~m}$. 29TQH (2).

118. Teverga. Cercanías Pto. Ventana. $1300 \mathrm{~m}$. 29TQH4371 (113).

119. Teverga. Pto. Ventana. 1700m. 29TQH4371 (1).

120. Proaza. Caranga de Arriba. 300m. 29TQH4188 (8).

121. Morcín. Majada de Fonfría. $1100 \mathrm{~m}$ 29TTN6091 (6).

122. Morcín. Fonfría. 1100m. 29TTN6091 (43).

123. Quirós. Sierro Espín. 1000m. 29TQH42 (1).

124. Quirós. Quirós. 300-1000m. 29TQH, 30TTN. (1).

125. Quirós. Vega de Duernas. 1150m. 29TQH4379 (2).

126. Varios concejos. Cordal de la Mesa. Puerto Ventana. $1340 \mathrm{~m}$. (1)

127. Mieres. Mieres. 300m. 29TTN79 (4).

128. Villaviciosa. Anayo. $300 \mathrm{~m}$. 30 TUP00 (1).

129. Piloña. Infiesto. $150 \mathrm{~m}$. 30TUP0802 (29).

130. Piloña. Pintueles. 150m. 30TUP0603 (17).

131. Parres. Mirador del Fito. 600m. 30TUP2212 (19).

132. Llanes. Rales. 20m. 30TUP4708 (22).

133. Llanes. Pendueles. 50m. 30TUP6706 (1).

134. Llanes. Buango. 5m. (2).

135. Lena. Pajares. 950m. 30TTN7466 (4).

136. Lena. Puerto de Pajares (E del parador). $1300 \mathrm{~m}$. 30TTN7563 (2).

137. Lena. Cercanías de la Colegiata de Arvás. $1400 \mathrm{~m}$. 30TTN7564 (2).

138. Lena. Valgrande. 1400m. 30TTN7462 (12).

139. Lena. Bosque de Valgrande. $900 \mathrm{~m}$. 30TTN76 (1).

140. Varios concejos. Parque Natural de Redes (7).

141. Caso. Monte Redes. 1100m. 30TUN17 (85).

142. Caso. Puerto de Tarna. $1500 \mathrm{~m}$. 30TUN 1972 (46).

143. Ponga. Entre Villarcazo y Cazo. $650 \mathrm{~m}$. 30TUN19 (3).

144. Ponga. Priescas. Río Ponga. 400m. 30TUN19 (1).

145. Ponga. Taranes. $600 \mathrm{~m}$. 30TUN2085 (21).

146. Ponga. Beleño (Los Bedules). $975 \mathrm{~m}$. 30TUN19 (2). 
147. Ponga. Beleño. $600 \mathrm{~m}$. 30TUN2484 (2).

148. Ponga. Viego. $950 \mathrm{~m}$. 30TUN2685 (3).

149. Ponga. Desfiladero de los Beyos. $510 \mathrm{~m}$. 30TUN3085 (3).

150. Cangas de Onís. Covadonga. $400 \mathrm{~m}$. 30TUN3397 (18).

151. Cangas de Onís. Vega de la Cueva. 1000m. 30 TUN3397 (1).

152. Cangas de Onís. Bosque de Pome. 800-1200m. 30 TUN39 (105).

153. Cangas de Onís. Lagos de Covadonga ( $7 \mathrm{~km} \mathrm{SE}$ de Covadonga). 1200m. 30TUN39 (9).

154. Cangas de Onís. Lago Enol (Covadonga). $1100 \mathrm{~m} .30 \mathrm{TUN} 3487$ (1).

155. Cangas de Onís. Covadonga (del lago La Ercina a Llaguiello). 1250m. 30TUN39 (5).

156. Cangas de Onís. Lago La Ercina (Covadonga). 1300m. 30TUN3992 (3).

157. Amieva. Valle del Dobra (majada de Les Curies, Picos de Europa). 1150m. 30TUN3487 (4).

158. Amieva. Río Dobra. Cerca del embalse la Jocica. 1000m. 30TUN3585 (4).

159. Cabrales. 8 km SSO Arenas de Cabrales. Río Cares. 250m. 30TUN49 (1).

160. Cabrales. Arenas de Cabrales. $300 \mathrm{~m}$. 30TUN5296 (5).

161. Cabrales. Bulnes (El Castillo). $700 \mathrm{~m}$. 30TUN5188 (1).

162. Cabrales. De Arenas a Trescares. $200 \mathrm{~m}$. 30TUN59 (7).

163. Cabrales. Picos de Hierro. 2400m. 30TUN58 (1).

164. Cabrales. Picos de Europa (E de Cangas de Onís, SE de Arenas de Cabrales). Cabrales. $1100 \mathrm{~m}$. 30TUN58 (1).

165. Peñamellera Alta. $0.7 \mathrm{Km} \mathrm{S}$ de Trescares. $250 \mathrm{~m}$. 30TUN6197 (13).

166. Ribadedeva. La Franca. 20m. 30TUP7205 (3).

Biótopos y comunidades sin localización geográfica precisa.

167. Numerosas en corrientes fluviales del Occidente. 20-1590m (1).

168. Sobre rocas costeras. Estadio Aerohalino (5). 169. Sobre rocas costeras. Estadio Higrohalino (6).

170. Sobre rocas básicas (20).

171. Sobre rocas ácidas (27).

172. Sobre suelos ácidos (22).

173. Sobre suelos básicos (14).
174. Alisedas del piso basal (15).

175. Encinares del piso basal (10).

176. Bosques frescos de avellanos y fresnos del piso basal (14).

177. Bosques mixtos de robles, castaños y abedules del piso basal (24).

178. Formaciones disclímax de pinos y eucaliptos del piso basal (14).

179. Hayedos y robledales del piso montano (71).

180. Abedulares del piso montano (5).

Estas localidades aparecen reflejadas en la figura 1, ordenadas de Oeste a Este y de Norte a Sur. Por otro lado, la definición de los pisos bioclimáticos se recoge en Díaz González y Fernández Prieto (1994)

\section{BIBLIOGRAFÍA}

ABBAYES, H. des -1945- Lichens d'Espagne récoltés de 1926 à 1935 par M. et Mme. Allorge. Rev. Bryol. Lichénol. 15(1-2):79-86.

BARRENO, E. y V.M. VÁZQUEZ -1981Coelocaulon crespoae Barreno y Vázquez sp. nova. Notas sobre la flora liquénica de brezales españoles. Lazaroa 3:235-246.

BOOM, P.G.G. van der \& A. GÓMEZ-BOLEA 1991- Contribution to the lichen flora of Spain. Nova Hedwigia 53(3-4):497-505.

BURGAZ, A.R. \& T. AHTI -1992-Contribution to the study of the genera Cladina and Cladonia in Spain. I. Nova Hedwigia 53(1-2):37-53.

BURGAZ, A.R. \& T. AHTI -1994- Contribution to the study of the genera Cladina and Cladonia in Spain. II. Nova Hedwigia 59(3-4):399-440.

CLAUZADE, G. \& C. ROUX -1985- Likenoj de Okcidenta Eùropo. Bull. Soc. Bot. Centre-Ouest, n. ser., num. Spec. 7:1-893.

COLMEIRO, M. -1889-Enumeración y revisión de las plantas de la Península Ibérica e Islas Baleares. Tomo V, Liquenes: 785-875. Madrid

CRESPO, A., E. BARRENO y V.M. VÁZQUEZ 1979- Buellia subcanescens (Physciaceae) en el norte de España. Lazaroa 1:139-142.

DEGELIUS, G. -1954- The lichen genus Collema in Europe: MORPHOLOGY, taxonomy and ecology. Symb. Bot. Upsal. 13(2):1-499.

DÍAZ GONZÁLEZ, T.E. y J.A. FERNÁNDEZ PRIETO -1994- El Paisaje Vegetal de Asturias. 
Guía de la Excursión. Itinera Geobot. 8:5-242.

DIZERBO, A.H. - 1956- Notes sur la flore marine de la côte atlantique de l'Espagne. Collect. Bot. 13(1):269-278.

EGEA, J.M. -1984- Contribución al conocimiento del género Caloplaca Th. Fr. en España: Especies saxícolas. Collect. Bot. 15:173-204.

JAUREGUI, J.A. - 1958- Viaje Botánico de Durieu por Asturias emprendido en el año 1835. Bol. Inst. Estud. Asturianos, Supl. Ci., 6:31-98.

KUROKAWA, S. -1962- A monograph of the genus Anaptychia. Nova Hedwigia (Beih.) 6. Weinheim.

LAZARO E IBIZA, B. -1898- Notas sobre algunos líquenes de España y Portugal. Actas Soc. Esp. Hist. Nat. (noviembre):180-186; (diciembre) 200-205.

LLIMONA, X.-1991-. Fongs i liquens. 5. In: X. Llimona (coor.) Historia Natural dels Països Catalans. Fundació Enciclopédia Catalana. Barcelona.

MARTÍNEZ, I. y A.R. BURGAZ - 1993-Estudio del género Peltigera en España peninsular. I. Cryptogamie, Bryol. Lichénol. 14(4):341-352.

MARTÍNEZ MORENO, M. I. -1999- Taxonomía del género Peltigera Willd. (Ascomycetes liquenizados) en la Penínula Ibérica y estudio de sus hongos liquenícolas. Ruizia 15:5-200.

NAVAS, L. -1901- Notas liquenológicas, II. El género Parmelia en España. Bol. R. Soc. Esp. Hist. Nat. 1(8):310-317.

NIMIS, P.L. -1993- The Lichens of Italy. Museo Regionale di Scienze Naturali. Torino.

OTTONSON, I. -1968- Usnea longissima Ach. Found in north-western Spain. Svensk Botanisk Tidskrift 62(4):515-520.

OZENDA, P. \& G. CLAUZADE-1970-. Les Lichens. Etude biologique et flore ilustrée. Ed. Masson et Cie. Paris.

POELT, J. -1969-Bestimmungsschlüssel Europäisher Flechten. Ed. J. Cramer. Lehrer.

PURVIS, O.W., B.J. COPPINS, D.L. HAWKSWORTH, P.W. JAMES \& D.M. MOORE (eds.) - 1992- The lichen flora of Great Britain and Ireland. The British Lichen Society. London.

RENOBALES, G. -1996- Contribución al conocimiento de los líquenes calcícolas del occidente de Vizcaya y parte oriental de Cantabria (N-España). Guineana 2:1-310.
SANTESSON, R. -1960- Lichenicolous fungi from Northern Spain. Svensk Botanisk Tidskrift 54(4):499-521.

TIMDAL, E. -1991- A monograph of the genus Toninia (Lecideaceae, Ascomycetes). Opera Botanica 110:1-137.

VÁZQUEZ, V.M. -1978- Notas liquenológicas IAportaciones al catálogo asturiano. Rev. Fac. Cienc. (Univ. Oviedo) 17:295-301.

VÁZQUEZ, V.M. -1981a- Líquenes epífitos como bioindicadores de la calidad del aire: Cartografía de la contaminación ambiental de la ría de Avilés. Bol. de información medioambiental del Excmo. Ayto. de Avilés 1:87-111.

VÁZQUEZ, V.M. -1981b- Los líquenes. In: T.E. Díaz Glez. et al. Enciclopedia temática de Asturias. I. Botánica: 96-139. Silverio Cañada ed. Gijón.

VÁZQUEZ, V.M. y A. CRESPO -1978- Catálogo de líquenes de Asturias. I. Epífitos. Acta Bot. Malacitana 4:11-26.

VITIKAINEN, O. -1980- Distribution patterns of European Peltigera. Biblioth. Lichenol. 25:423-426.

Aceptado para su publicación en abril de 2000

Dirección de los autores. Fernando DE LA TORRE FDEZ. INDUROT - Institu to de Recursos Naturales y Ordenación del Territorio. Universidad de Oviedo. Independencia 13. 33.004 - Oviedo (España). Email: delatorre@indurot.uniovi.es; $M^{\mathrm{a}}$ Carmen FERNÁNDEZ ORDÓÑEZ. Unidad de Botánica. Departamento de Biología de Organismos y Sistemas. Universidad de Oviedo. Catedrático Rodrigo Uría s/n. 33.006 - Oviedo (España). Email: mcfernan@correo.uniovi.es 